\title{
Versorgungsstrukturgesetz und Gesetz zur Neuordnung des Arzneimittelmarktes
}

\section{Christian Dierks}

Dierks + Bohle, Rechtsanwälte, Berlin, Deutschland

Durch das Versorgungsstrukturgesetz haben sich vor allem in der ambulanten vertragsärztlichen Tätigkeit Änderungen ergeben, die zum Januar 2013 in Kraft getreten sind:

- Bei der Nachbesetzung von Vertragsarztsitzen kann der Zulassungsausschuss künftig die Nachbesetzung ablehnen, wenn sie aus Gründen der Versorgung nicht erforderlich ist. Die kassenärztliche Vereinigung muss dann eine Entschädigung an den Abgeber in Höhe des Verkehrswerts zahlen.

- Die Residenzpflicht wird abgeschafft.

- Nebentätigkeiten sind künftig grundsätzlich zulässig, wenn ausreichende Sprechstundenzeit angeboten werden kann.

- Angestellte Arztsitze können künftig in Vertragsarztsitze umgewandelt werden. Sie können auch veräußert werden, wenn sie nicht mehr benötigt werden.

- Die Verlegung eines Vertragsarztsitzes darf künftig nur genehmigt werden, wenn Gründe der vertragsärztlichen Versorgung nicht entgegenstehen.

- Weitere wichtige Veränderungen betreffen die Einführung der ambulanten spezialfachärztlichen Versorgung (ASV), die regionalisierte Honorarverteilung und die neu strukturierte Bedarfsplanung. Hierzu werden vom Gemeinsamen Bundesausschuss (G-BA) und der Kassenärztlichen Bundesvereinigung im Laufe des Jahres weitere Regelungen veröffentlicht werden.

Mit dem Gesetz zur Neuordnung des Arzneimittelmarktes (AMNOG) wurde zu Beginn des Jahres 2011 ein Mechanismus zur Bewertung des Zusatznutzens von neuen Arzneimitteln geschaffen. Der G-BA bewertet hierzu das vom Hersteller vorgelegte Dossier nach Prüfung durch das Institut für Qualität und Wirtschaftlichkeit im Gesundheitswesen (IQWiG). Auf der Basis dieser Bewertung werden Preise vereinbart, die nach der gesetzlichen Definition als wirtschaftlich gelten. Daraus ergeben sich für die vertragsärztliche Versorgung zwei Konsequenzen:

- Zum einen kann die Verordnung der so bewerteten und mit wirtschaftlichen Preisen versehenen Substanzen in einem Wirtschaftlichkeitsprüfverfahren nicht beanstandet werden, wenn Indikation und Dosierung eingehalten wurden. Entsprechende Details hierzu finden sich auf der Website des Spitzenverbandes Bund der Krankenkassen. Im Regelfall ist die Verordnung dieser innovativen Arzneimittel von den Prüfgremien als wirtschaftlich zu akzeptieren.

- Andererseits ist eine Verordnung im ersten Jahr nach der Zulassung nicht deswegen unwirtschaftlich, weil die Gesetzliche
Krankenversicherung noch den Preis entrichten muss, den der Hersteller selbst festgesetzt hat. Es liegt im Wesen dieses Systems, dass die Zeit bis zur Vereinbarung des Erstattungspreises als legitime Einführungsphase keiner besonderen Wirtschaftlichkeitsprüfung ausgesetzt ist. Regresse können also nicht deshalb verhängt werden, weil die Kosten des Arzneimittels in diesen ersten Monaten noch über dem später festgesetzten Erstattungspreis lagen.

Für Ipilimumab $\left(\right.$ Yervoy $^{\circledR}$ ) wurde das Verfahren zur frühen Nutzenbewertung inzwischen abgeschlossen. Das Arzneimittel ist zugelassen zur Behandlung von vorbehandelten erwachsenen Patienten mit fortgeschrittenen (nicht resezierbaren oder metastasierten) Melanomen. Für Yervoy ${ }^{\circledR}$ hat die Bewertung des G-BA einen Hinweis auf einen beträchtlichen Zusatznutzen ergeben. Auf dieser Basis haben sich der Spitzenverband Bund und der Hersteller des Arzneimittels ohne Schiedsstelle auf einen Erstattungsbetrag geeinigt. Die Vereinbarung gilt rückwirkend ab dem 01.08.2012. Der neue Preis gilt nach $\S 130$ b SGB V (Sozialgesetzbuch Fünftes Buch) als wirtschaftlicher Erstattungsbetrag. Vereinbarungen dieser Art sollen vorsehen, dass Verordnungen des Arzneimittels von der Prüfungsstelle als Praxisbesonderheiten anerkannt werden, wenn der Arzt bei der Verordnung im Einzelfall die dafür vereinbarten Anforderungen an die Verordnung eingehalten hat. Diese Anforderungen sind in den Programmen zur Verordnung von Arzneimitteln, die von den kassenärztlichen Vereinigungen veröffentlicht werden, zu hinterlegen. Hierzu wird noch eine Veröffentlichung auf der Website des Spitzenverbandes Bund der Gesetzlichen Krankenversicherung erwartet. Diese wird ihre entsprechende Abbildung in den Prüfvereinbarungen der einzelnen kassenärztlichen Vereinigungen finden. Entsprechend gelistete Arzneimittel gelten dann als Praxisbesonderheit. Insgesamt wird das Gesetz dazu führen, dass durch die Bewertung des Zusatznutzens und die Vereinbarung eines Erstattungspreises die Verordnung von innovativen Arzneimitteln zukünftig regresssicher erfolgen kann.

\section{Disclosure Statement}

Der Autor war Referent beim «3. Interdisziplinären Expertenforum Hautkrebs», das von Bristol-Myers Squibb veranstaltet und unterstützt wurde.

\begin{tabular}{ll}
\hline KARGER & @ 2013 S. Karger GmbH, Freiburg \\
0378-584X/13/3616-0012\$38.00/0 \\
$\begin{array}{l}\text { Fax +49 761 4520714 } \\
\text { Information@Karger.com } \\
\text { www.karger.com }\end{array}$ & $\begin{array}{l}\text { Accessible online at: } \\
\text { www.karger.com/onk }\end{array}$
\end{tabular}

